\title{
Finite Horizons, Political Economy, and Growth
}

\author{
James A. Kahn and Jong-Soo Lim* \\ March 2000 \\ Forthcoming, Review of Economic Dynamics
}

*Kahn, Domestic R esearch, Federal Reserve Bank of New York, james.kahn@ny.frb.org; Lim, Department of International Trade, Kwangwoon University, Seoul, Republic of K orea, jslim@daisy.kwangwoon.ac.kr. We thank seminar participants at the University of R ochester and conference participants at the Federal R eserve Bank of M inneapolis Conference on Economics and Politics for helpful discussions. The views expressed are those of the authors and do not necessarily reflect the views of the Federal Reserve Bank of $\mathrm{New}$ York or the Federal R eserve System. 


\begin{abstract}
A bstract
This paper analyzes the political economy of growth when agents and the government have finite horizons and equilibrium growth is inefficient. A "representative" government (i.e. one whose preferences reflect those of its constituents) endowed merely with the ability to tax and transfer can improve somewhat on the market allocation, but cannot achieve first-best growth. Efficiency requires in addition the ability to bind future governments. We argue that this ability is related to political stability, and provide empirical evidence that stability and growth-related policies (namely education) are meaningfully related.
\end{abstract}


Recent research on economic growth has found a negative relationship between political instability and growth. ${ }^{1}$ If causal, this correlation could come from either the direct impact of instability (the impact of wars or revolutions, for example, on the physical and human capital stocks), or from the indirect impact through its effect on government decision-making. Instability could give rise to shortsightedness on the part of policymakers, either because they do not expect to remain in power to reap whatever rewards come from better policies, or because the instability itself prevents them from establishing long-lived institutions that could facilitate more efficient policies by binding future governments.

In this paper we explore the indirect effect of political instability on growth through its impact on political decisions. We begin with a model of the political economy of growth in which the finite horizons of economic agents, coupled with the inability of current governments to bind future policymakers, leads to inefficiently low growth. The overlapping generations framework considered in this paper requires intervention to achieve optimal growth. This is for the usual reason in such models: Intergenerational trade is not possible, so mutually beneficial exchanges between generations fail to take place. Later generations cannot reward earlier generations for their investments in human capital, so everyone underinvests relative to a world in which a farsighted or long-lived planner can bring about efficiency. We then examine whether in such a setting a realistic political system is likely to deliver anything close to optimal growth.

Specifically, we argue that it is realistic to consider a policymaker whose objective reflects that of the population for which he is making decisions. In contrast to the infinite horizon planner, the more realistic scenario requires the ability to bind future policymakers' decisions in order to achieve efficiency. We further argue that political instability precludes the sort of institutions that can effectively bring about precommitment as a substitute for intergenerational exchange. Without such precommitment (or its equivalent in the form of "trigger strategy" equilibria), the sequence of finite-horizon planners will enact policies that, while improving on the 
market equilibrium, exhibit inefficiently low growth.

The model in the first part of the paper is a simple endogenous growth model in which the engine of growth is the accumulation of knowledge. The choice of education is suggested by the fact (e.g. Psacharopoulos, 1973) that less developed countries have a significantly higher return to human capital accumulation (e.g. the return to schooling) than developed countries, suggesting that they are systematically underinvesting in that resource. We assume that a higher level of knowledge attained by one generation reduces the cost of attaining that same level by the next (the usual "standing on the shoulders of giants" story), an externality which has the consequence that the laissez-faire equilibrium growth rate is inefficient. We characterize the set of Pareto efficient accumulation paths and find that there is a continuum of efficient growth rate-interest rate combinations, the choice among which depends on the social discount rate. Competitive equilibrium with subsidized or mandated accumulation of knowledge may give rise to a Pareto efficient steady state, though for some parameters dynamic efficiency requires intergenerational redistribution.

We then go on to the main topic of the paper, which is how a planner whose objective reflects the finite horizons of his constituents would choose policies, and what the outcome looks like. We assume that each period the current government maximizes a weighted sum of utilities of those currently alive. Policy decisions are modeled as the outcome of a non-cooperative dynamic Stackelberg game: Each period the government selects a policy that takes into account the effect (through state variables) on subsequent policy decisions (and hence on the welfare of the current young generation). Numerical methods are used to compute Markov equilibrium policy rules (defined as fixed points in the space of possible functions) under specific parametric assumptions. The outcome is generally inefficient, and for plausible parameters exhibits a growth rate that is substantially below the efficient rate, while still an improvement over laissez-faire.

We argue finally that the ability to get beyond the Markov equilibrium toward efficient growth requires political stability, i.e. the ability to precommit through the 
establishment of institutions that outlive the current government and bind future decisionmakers. We offer empirical evidence to show that political instability is associated with lower levels of education (and government support thereof), even after controlling for income levels.

The first section of the paper sets up the framework, an overlapping generations model with endogenous growth and contrasts the competitive equilibrium with the efficient allocation. Section 2 constructs the political equilibrium and provides numerical solutions. Section 3 offers empirical evidence on policy and political instability. Section 4 concludes.

\section{The Model Economy}

The model adapts the standard neoclassical overlapping generations model of capital accumulation to incorporate endogenous growth. In a sense it represents a cross between Diamond (1965) and Uzawa (1965). ${ }^{2}$ Each generation allocates time between labor and the accumulation of knowledge. Output depends on physical capital and effective labor, and exhibits constant returns to scale. Knowledge is passed (at least to some degree) from one generation on to the next, along with physical capital. We assume only that a higher level of knowledge attained in one generation makes it less costly for the next generation to attain the same level. Thus the fact that the Wright brothers' generation discovered how to make airplanes fly did not mean that the next generation was born with this knowledge, only that it could attain that knowledge more easily, and without fully rewarding its predecessors (hence the externality).

We assume that within each period knowledge accumulated by an individual translates directly into his human capital, without any external spillovers. Hence in what follows we will speak of knowledge and human capital interchangeably. There is, however, an intergenerational externality, owing to the nonexcludability of knowledge across generations. That is, the older generation cannot sell its stock of knowledge to the young generation. In the model this is simply assumed, but even if it were tech- 
nically possible to make the stock of knowledge excludable, the young have nothing to offer the old in exchange for it. ${ }^{3}$

Individuals live for two periods. All individuals within each cohort are identical. In their first period they allocate time between labor and accumulation of knowledge. We will refer to the time spent on human capital accumulation as "schooling", though a more apt interpretation is the share of flexible resources (in this case time) that productive individuals allocate to increasing their knowledge rather than producing. The wage they earn for labor depends on their accumulated human capital. They allocate their wage income in the first period between consumption when young and consumption when old. When old, individuals consume their savings plus interest.

Each individual solves the problem

$$
\operatorname{Max} u\left(C_{1 t}\right)+\beta u\left(C_{2 t+1}\right)
$$

subject to

$$
\begin{aligned}
C_{1 t}+C_{2 t+1} /\left(1+r_{t+1}\right) & =w_{t} H_{t} \ell_{t} \\
H_{t} & =g\left(\ell_{t}\right) \bar{H}_{t-1}
\end{aligned}
$$

where $w_{t}$ is the wage per unit of human capital, $H_{t}$ is the individual's human capital stock, $\bar{H}_{t-1}$ is the average human capital level of the previous generation, $r_{t+1}$ is the interest rate, and $\ell_{t} \in[0,1]$ is the proportion of time allocated to labor. The remaining time $1-\ell_{t}$ is allocated to human capital accumulation. We assume that $d g / d \ell \leq 0$, that $g(0)<\infty, g(1) \geq 0$, and that $d u / d c>0, d^{2} u / d c^{2}<0$. Since all individuals within a cohort are assumed to be identical, we know that $H_{t}=\bar{H}_{t}$, so we will drop the distinction for the remainder of the paper. Also, we will frequently use subscripts 1 or 2 on functions to denote the derivative with respect to the first or second argument of a function (e.g. $\left.g_{1}(\ell) \equiv d g / d \ell, u_{11}(c) \equiv d^{2} u / d c^{2}\right)$. The ' symbol will be reserved to denote "one period ahead" in Section 2. 
The first order conditions for the individual's maximization problem are

$$
u_{1}\left(C_{1 t}\right)=\beta\left(1+r_{t+1}\right) u_{1}\left(C_{2 t+1}\right)
$$

and

$$
\ell_{t} g_{1}\left(\ell_{t}\right)+g\left(\ell_{t}\right)=0
$$

assuming interior solutions. Thus the individual simply chooses $\ell_{t}$ to maximize his earnings $w_{t} \ell_{t} H_{t}$, given (2). The solution to (4) - and consequently the equilibrium growth rate - is independent of $K_{t}$ and $H_{t-1}$.

Output is produced from a constant returns to scale production technology $F\left(K_{t}, \ell_{t} H_{t}\right)$. Here we normalize the population to unity and ignore population growth (which does not change anything substantive) Competitive firms maximize profits, taking the wage and interest rate as given. Profit maximization implies

$$
F_{1}\left(K_{t}, \ell_{t} H_{t}\right)=r_{t},
$$

and

$$
F_{2}\left(K_{t}, \ell_{t} H_{t}\right)=w_{t}
$$

Thus the model is a straight generalization of Diamond's (1965) model. To reproduce that model we would set $g(\ell)=1$. The equilibrium value of $\ell$ would be 1 , the level of human capital would be fixed, and all of Diamond's results would follow.

In order to make the generalization interesting, we make one regularity assumption on $g(\ell)$. First define $\ell^{*}=\underset{\ell}{\arg \max } \ell g(\ell)$. Then we assume

A 1: $\ell^{*}<1$.

The assumption that $g(0)<\infty$ already rules out $\ell^{*}=0$, so $\mathrm{A} 1$ guarantees an interior solution for $\ell$.

Equilibrium requires (3)-(6) and

$$
C_{1 t}+C_{2 t}+K_{t+1}=F\left(K_{t}, H_{t} \ell_{t}\right)+K_{t}
$$


or

$$
C_{1 t}+C_{2 t}=F\left(K_{t}, H_{t} \ell_{t}\right)-\left(K_{t+1}-K_{t}\right)
$$

where $H_{t-1}$ and $K_{t}$ are predetermined state variables for period $t$. Since $\ell^{*}$ is independent of the state variables, we can fix $g(\ell)$ and $\ell \forall t$. The equilibrium conditions imply that

$$
\begin{gathered}
C_{2 t}=K_{t}\left(1+r_{t}\right) \\
C_{1 t}=F\left(K_{t}, H_{t} \ell_{t}\right)-K_{t+1}-K_{t} r_{t} \\
u_{1}\left(C_{1 t}\right)=\beta\left(1+r_{t+1}\right) u_{1}\left(C_{2 t+1}\right)
\end{gathered}
$$

Given $H_{t-1}$ and $K_{t}$, we have $H_{t}=g\left(\ell^{*}\right) H_{t-1}$, and equations (5)-(6), (9)-(11) determine $C_{1 t}, C_{2 t}, K_{t+1}, w_{t}$, and $r_{t}$.

We will focus on balanced growth steady states in which $k_{t} \equiv K_{t} /\left(H_{t} \ell_{t}\right)$ is constant, under the assumption

$$
\text { A 2: } u(C)=\left\{\begin{array}{l}
C^{1-1 / \sigma} /(1-1 / \sigma), \text { if } \sigma \neq 1 \\
\log (C) \text { otherwise }
\end{array}\right.
$$

where $\sigma>0$. In such a steady state, $K, H, C_{1}$, and $C_{2}$ all grow at the rate $g(\ell)-1$. Conditional on $\ell^{*}$, analysis of competitive equilibrium proceeds entirely as in Diamond (1965), though with a fixed growth rate $g\left(\ell^{*}\right)-1$. In particular, the equilibrium may or may not be dynamically efficient. We shall see shortly, however, that the competitive outcome is always Pareto inefficient. We first analyze the problem of a planner with a fixed social discount rate.

\subsection{A Social Planner's Problem}

We first consider the solution of an infinitely lived social planner who discounts the utility of generations at rate $\rho>0 .{ }^{4}$ At time 1 he chooses a path $\left\{C_{1 t}, C_{2 t}, \ell_{t}\right\}$ from $t=1$ to $\infty$ to solve the problem

$$
\operatorname{Max} \sum_{t=1}^{\infty}(1+\rho)^{-t+1}\left[u\left(C_{1 t}\right)+\beta u\left(C_{2 t+1}\right)\right]
$$


subject to

$$
\begin{gathered}
C_{1 t}+C_{2 t}+K_{t+1}=F\left(K_{t}, H_{t} \ell_{t}\right)+K_{t}, \\
H_{t}=H_{t-1} g\left(\ell_{t}\right)
\end{gathered}
$$

given $K_{1}, H_{0}$, and $c_{21}$.

We can set up the following Lagrangian:

$$
\begin{aligned}
\mathcal{L}= & \sum_{t=1}^{\infty}(1+\rho)^{-t+1}\left(\left[u\left(C_{1 t}\right)+\beta u\left(C_{2 t+1}\right)\right]+\right. \\
& \lambda_{t}\left[F\left(K_{t}, H_{t} \ell_{t}\right)+K_{t}-C_{1 t}-C_{2 t}-K_{t+1}\right]- \\
& \left.\mu_{t}\left[H_{t}-H_{t-1} g\left(\ell_{t}\right)\right]\right)
\end{aligned}
$$

where $\lambda_{t}$ and $\mu_{t}$ are multipliers associated with the two transition equations. The first order conditions for the solution of the optimization problem in $\left\{K_{t+1}, H_{t}, c_{1 t}, c_{2 t}, \ell_{t}, \lambda_{t}, \mu_{t}\right\}$ are

$$
\begin{aligned}
u_{1}\left(C_{1 t}\right) & =\lambda_{t} \\
\beta u_{1}\left(C_{2 t}\right) & =\lambda_{t} /(1+\rho) \\
\lambda_{t} H_{t} F_{2}\left(K_{t}, H_{t} \ell_{t}\right) & =-\mu_{t} g_{1}\left(\ell_{t}\right) H_{t-1} \\
\lambda_{t} \ell_{t} F_{2}\left(K_{t}, H_{t} \ell_{t}\right) & =\mu_{t}-\mu_{t+1} g\left(\ell_{t+1}\right) /(1+\rho) \\
\lambda_{t}\left[1+F_{1}\left(K_{t}, H_{t} \ell_{t}\right)\right] & =\lambda_{t-1}(1+\rho)
\end{aligned}
$$

along with the two constraints (12) and (13).

We will focus only on the optimal balanced growth steady state in which $r$ and $\ell$ are constant (and hence $K, C_{1}, C_{2}$, and $H$ all grow at rate $g(\ell)$ ).. First, (15) and (16) imply that the growth rates of $C_{1 t}$ and $C_{2 t}$ are the same in the steady state, as one would expect. Also, the homogeneity of $F$ implies that the rate of growth of consumption is equal to the rate of growth of human capital. With the CES utility 
function assumed above, we have

$$
g(\ell)^{1 / \sigma}=\lambda_{t} / \lambda_{t+1} .
$$

Equation (19) implies that

$$
\lambda_{t} / \lambda_{t+1}=\left[1+F_{1}(K, H \ell)\right] /(1+\rho) .
$$

Hence from equation (17) and (20) we have $\mu_{t+1} / \mu_{t}=g(\ell)^{-1 / \sigma}$, which, after some straightforward substitutions, yields:

$$
1+g_{1}(\ell) \ell / g(\ell)=g(\ell)^{1-1 / \sigma} /(1+\rho) .
$$

Finally, (20) and (21) imply

$$
1+F_{1}\left(K_{t}, H_{t} \ell_{t}\right)=(1+\rho) g(\ell)^{1 / \sigma} .
$$

Equations (22) and (23) determine the planner's choice of $\ell$, denoted $\ell_{p}$, which in turn determines the optimal growth rate $g(\ell)$. While (23) is a standard $M R S=M R T$ condition, equation (22) equates the marginal foregone output from additional work to the discounted value of the resulting increased output the following period, in utility terms.

We can compare (22) with the equilibrium condition implied by $(4), 1+g_{1}(\ell) \ell / g(\ell)=$ 0 . The two conditions coincide as $\rho \rightarrow \infty$, as one might expect, because then $F_{1}(K, H \ell) \rightarrow \infty$ by (23). The optimal and equilibrium growth rates also coincide in the limit as $\sigma$, the intertemporal elasticity of substitution, goes to zero. As $\sigma$ increases the optimal growth rate increases as well. Except for the extreme cases, the planner's optimal $\ell$ is lower than the equilibrium $\ell$, which means that the optimal growth rate generally exceeds the equilibrium growth rate for any $\rho<\infty$.

The social planner's optimum yields a particular set of Pareto efficient allocations associated with different social discount rates, but as is well known from the work 
of Diamond (1965), Cass (1972), and others, the fundamental theorems of welfare economics do not apply to these economies. The competitive equilibrium need not be Pareto efficient, and the Pareto optima given by the planner's problem may not be achievable by decentralized equilibrium.

The work of Cass (1972) and others suggests that a sufficient condition for dynamic efficiency of the path $\left\{K_{t}\right\}$, conditional on $\left\{\ell_{t}\right\}$, is that

$$
\lim _{t \rightarrow \infty} \prod_{s=0}^{t}\left[1+F_{1}\left(K_{s}, H_{s} \ell_{s}\right)\right] / g\left(\ell_{s}\right)>0
$$

In a steady state this condition translates into

$$
1+F_{1}(K, H \ell) \geq g(\ell)
$$

which, as we have seen, is satisfied by the planner's optimum. If for convenience we use $c_{1}, c_{2}$, and $k$ to refer to the corresponding upper-case variables divided by $H \ell$ (so that the steady state can be expressed in terms of quantities that are constant in the steady state), the resource constraint (12) $C_{1 t}+C_{2 t}+K_{t+1}=F\left(K_{t}, H_{t} \ell_{t}\right)+K_{t}$, becomes

$$
c_{1}+c_{2}=f(k)-[\ell g(\ell)-1] k .
$$

where $f(k)=F(k, 1)$. If $1+r$ were less than $g(\ell)$, then reducing $K$ (i.e. reducing $k$ for a given $\ell$ ) would increase steady state consumption, a contradiction of efficiency.

We can combine conditions (22) and (23) to get an expression that relates optimal growth to the marginal product of capital that depends only on technology:

$$
1+\ell g_{1}(\ell) / g(\ell)=g(\ell) /\left[1+f_{1}(k)\right]
$$

Equilibrium conditions will determine $k$, and these will generally depend on preferences, population growth, and government policies. Note that (27) implies

$$
1+f_{1}(k)>g(\ell)
$$


for any steady state that has positive production. That is, in any efficient steady state with positive production, $k$ must be strictly smaller than that which maximizes consumption per worker. This is because $1+f_{1}(k)=g(\ell)$ and (27) together would imply $\ell g_{1}(\ell) / g(\ell)=0$, or $\ell=0$. Consequently if $\ell$ is chosen efficiently, steady state dynamic efficiency in $k$ is assured.

How could a Pareto efficient outcome be implemented? Essentially all that would be necessary is some mechanism to control $\ell$, e.g. "mandatory schooling", plus in some instances the ability to make intergenerational transfers. Together with competitive labor and goods markets, these suffice to bring about a Pareto efficient steady state. Note, however, that the equilibrium $k$ is normally increasing in $\ell$ (i.e. decreasing in the growth rate). This is because a higher growth rate causes reduced savings.

We can let $\psi(\ell)$ denote the competitive equilibrium steady state value of $k$ as a function of an exogenously imposed $\ell$. Let $\zeta(\ell)$ denote the steady state value of $k$ as a function of $\ell$ that satisfies the efficiency condition (27). With $\psi(\ell)$ upward-sloping, and $\zeta(\ell)$ downward sloping, the intersection yields the unique efficient steady state $(\ell, k)$ under the assumption that a planner chooses the optimal $\ell$ while $k$ is determined competitively.

Example: Suppose $f(k)=A k^{\alpha}$, and again assume $u(C)=\log (C), g(\ell)=G(1-$ $\left.\ell^{\nu}\right)^{\xi}$, where $\nu>1, \xi<1$. Figure 1 displays the competitive equilibrium for the parameters $A=6, \alpha=.3, G=2, \nu=2$, and $\xi=.5\left(\right.$ so $\left.g(\ell)=2 \sqrt{1-\ell^{2}}\right)$. The efficient $\ell$, denoted $\ell_{e}$, is approximately 0.57 , which corresponds to $g\left(\ell_{e}\right)=1.72$. With a 30-year time period this would correspond to 1.82 percent annual growth. Laissezfaire $\ell^{*}$, on the other hand is 0.71 . We have $g\left(\ell^{*}\right)=1.41$, or 1.16 percent annual growth.

To summarize, competitive equilibrium in this model has inefficiently low growth. There is a range of efficient growth rate-interest rate combinations that a planner can achieve with some combination of mandatory schooling and intergenerational transfers. The remainder of the paper will drop the assumption that governments necessarily implement efficiency, and replace it with the assumption that governments 
maximize the welfare of the people currently alive, without being able to bind future governments.

\section{Political Economy}

For the remainder of the paper the political decisionmaker is assumed each period to maximize a weighted sum of the utilities of those currently alive, taking into account the fact that the same decision process will take place in the next period, and that the choice today will influence next period's choice through its influence on the state variables of the economy. Thus political choice is depicted as a dynamic Stackelberg game between governments at different time periods. The decision problem within each period is treated like a bargaining problem, with the government selecting some point on the contract curve. ${ }^{5}$ A solution technique is developed to solve for the Markov Perfect equilibrium of this game as applied to the model from the first part of the paper. We assume that the political system chooses $\ell$ and the size and direction of intergenerational transfers.

In general the inability to bind subsequent governments results in inefficiency. It turns out that the government improves upon the competitive equilibrium, but does not achieve Pareto efficiency. There exists a steady state policy that would make everyone better off by increasing growth (at the expense of current output) and increasing transfers to the old. That policy is not selected, however, because each government cannot bind the next government to carry out the transfer (from the next generation's young to their elders) that results in the current generation remaining at least as well off as under the Markov equilibrium outcome.

The model economy is the same as in Section 1 except that it will now incorporate an explicit policy of lump-sum intergenerational transfers. The consumption and savings decisions of individuals are determined in a competitive equilibrium in which each individual takes the political decision as given. The political decision, however, takes into account its effect on individual decision-making, and hence on the political 
decision of the next period. We introduce at this time a minor refinement in notation: $\bar{K}_{t}$ denotes the aggregate quantity of capital at date $t$ (which individuals view as exogenous), while $K_{t}$ denotes the value that a representative individual chooses. Of course in equilibrium the two quantities are identical. Hence the individual's budget constraint is

$$
C_{1 t}+C_{2 t+1} /\left(1+r_{t+1}\right)=w_{t} H_{t} \ell_{t}-\tau_{t} H_{t}+\tau_{t+1} H_{t+1} /\left(1+r_{t+1}\right)
$$

where $w_{t}$ is the wage per unit of effective labor, and $\tau_{t}$ is the politically-determined lump-sum transfer (scaled by the level of the economy so that $\tau$ will be constant in a balanced-growth steady state) from cohort $t$ to cohort $t-1$ at date $t$.

The first order conditions for the individual's maximization problem are as before:

$$
u_{1}\left(C_{1 t}\right)=\beta\left(1+r_{t+1}\right) u_{1}\left(C_{2 t+1}\right)
$$

and the budget constraint (29). Equilibrium still requires (12) and (13), the equations that give the evolution of $K_{t}$ and $H_{t}$. For a given time path of the policy variables $\tau_{t}$ and $\ell_{t}$, the model can be solved for the equilibrium path of $K_{t}, C_{1 t}, C_{2 t}, w_{t}$, and $r_{t}$.

The political system at time $t$ is assumed to choose $\tau_{t}$ and $\ell_{t}$ to solve

$$
\operatorname{Max}_{\ell t, \tau_{\mathrm{t}}} \theta \beta u\left(C_{2 t}\right)+(1-\theta)\left[u\left(C_{1 t}\right)+\beta u\left(C_{2 t+1}\right)\right]
$$

subject to

$$
\begin{gathered}
C_{1 t}=H_{t} \ell_{t} w_{t}-K_{t+1}-\tau_{t} H_{t} \\
C_{2 t}=K_{t}\left(1+r_{t}\right)+H_{t} \tau_{t}
\end{gathered}
$$

given $\bar{K}_{t}$ and $\bar{H}_{t-1}$, with $r_{t}=F_{1}\left(\bar{K}_{t}, H_{t} \ell_{t}\right)$ and $w_{t}=F_{2}\left(\bar{K}_{t}, H_{t} \ell_{t}\right)$, and knowing that at $t+1$ the same decision process will determine $\ell_{t+1}$ and $\tau_{t+1}{ }^{6}$

Thus the political decision at $t$ takes into account its effect on all future political decisions, since the decision at $t+1$ takes into account its effect on $t+2$, and so forth. 
The parameter $\theta$ represents a welfare weight on the old relative to the young that is assumed for simplicity to be constant from one period to the next. Note that while $\theta$ could be related to a generational discount rate like $\rho$ (e.g. $\theta /(1-\theta)=1+\rho)$, there is no reason a priori to restrict $\theta$ beyond requiring that it lie in the unit interval. ${ }^{7}$

The result is a decision for $\left(\tau_{t}, \ell_{t}\right)$ that should only depend directly on $\bar{K}_{t}, H_{t-1}$ and next period's decision rule $\left(\tau_{t+1}, \ell_{t+1}\right) \equiv \Gamma_{t+1}\left(\bar{K}_{t+1}, H_{t} ; \ldots\right)$. Consequently we have $\Gamma_{t}\left(\bar{K}_{t}, H_{t-1} ; \Gamma_{t+1}\left(\bar{K}_{t+1}, H_{t} ; \Gamma_{t+2}\left(\bar{K}_{t+2}, H_{t+1} ; \ldots\right), \ldots\right)\right)$. But in a symmetric Markov equilibrium the state of the system at entering time $t$ is fully described by $\bar{K}_{t}$ and $H_{t-1}$, so the equilibrium strategy can be described simply as $\Gamma\left(\bar{K}, H_{-1}\right)$. Thus we are looking for the following:

Political equilibrium: A function $\Gamma\left(\bar{K}, H_{-1}\right)$ such that if the government at $t+1$ chooses $\left(\tau_{t+1}, \ell_{t+1}\right)$ according to $\Gamma\left(\bar{K}_{t+1}, H_{t}\right)$, then the solution to (P3) is $\left(\tau_{t}, \ell_{t}\right)=$ $\Gamma\left(\bar{K}_{t}, H_{t-1}\right)$

Actually finding an equilibrium policy rule remains a difficult task. It is possible in general only to characterize equilibrium sufficiently so that numerical techniques can find a solution under specific parametric assumptions. The results are suggestive of more general conclusions, and in any case can be compared to the "cooperative" solution of a longer- or infinitely-lived social planner. We do not address the questions of existence and uniqueness of equilibrium.

\subsection{M odel Solution}

To economize on notation, we will let primed (') variables refer to one period ahead, and subscript ${ }_{-1}$ refer to lagged variables. To solve the model we start at an arbitrary time $t$ with an arbitrary policy rule $\Gamma^{\prime}\left(\bar{K}^{\prime}, H\right)$ specified for the next period. This generates first-order conditions that characterize a policy rule $\Gamma\left(\bar{K}, H_{-1} \mid \Gamma^{\prime}\left(\bar{K}^{\prime}, H\right)\right)$. This process can be repeated until the function so generated converges to a rule $\Gamma^{*}\left(\bar{K}, H_{-1}\right)$. The iteration process should not be thought of as dynamic convergence to a "steady state" $\Gamma^{*}$ function; it is just an expositional method for characterizing the equilibrium. The function so computed is valid globally, not just in the steady 
state.

Although both $\bar{K}$ and $H_{-1}$ are state variables, in fact the model has been formulated in such a way that the two policy instruments $\ell$ and $\tau$ will only depend on the ratio $\bar{K} / H_{-1}$. This is because of the homotheticity built into both preferences and technology (and the definition of $\tau$ as a fraction of $H$ ). In addition, for convenience we will define $k \equiv K /(H \ell)=K /\left(H_{-1} g(\ell) \ell\right)$ (with the analogous definition for $\bar{k}$ ). Even though $\bar{k}$ so defined is not, strictly speaking, a state variable, there is no loss in generality or accuracy in defining policy rules in terms of $\bar{k}$, and there is a large gain in simplicity. ${ }^{8}$ Therefore we will use $\ell(\bar{k})$ and $\tau(\bar{k})$ in lieu of $\Gamma\left(\bar{K}, H_{-1}\right)$. So we will solve the problem

$$
\underset{\ell, \tau}{\operatorname{Max}} \theta \beta u\left(C_{2}\right)+(1-\theta)\left\{u\left(C_{1}\right)+\beta u\left(C_{2}^{\prime}\right)\right\}
$$

subject to

$$
\begin{aligned}
& C_{1} / H_{-1}=g(\ell)\left[\ell w-g\left(\ell^{\prime}\right) \ell^{\prime} k^{\prime}-\tau\right] \\
& C_{2} / H_{-1}=g(\ell)[\ell k(1+r)+\tau]
\end{aligned}
$$

given $\bar{K}$ and $H_{-1}$, with $k=K /(H \ell)$, and $\bar{k}=\bar{K} /(H \ell), r=f_{1}(\bar{k}), w=f(\bar{k})-r \bar{k}$, and given arbitrary (but differentiable) one-period-ahead policy rules $\tau^{\prime}\left(\bar{k}^{\prime}\right), \ell^{\prime}\left(\bar{k}^{\prime}\right) .{ }^{9}$

Note that (30)-(32) determine a function $k\left(\tau, \tau^{\prime}\left(\bar{k}^{\prime}\right), \ell, \ell^{\prime}\left(\bar{k}^{\prime}\right)\right)$. That is, individuals choose savings taking policy variables as given. But they know that $\bar{k}^{\prime}=k^{\prime}$; hence if $\tau$ or $\ell$ change, with perfect foresight consumers take account of the effect on $\tau^{\prime}$ through the effect on $\bar{k}^{\prime}$. So to get, for example, the total effect of a change in $\tau$ on $k^{\prime}$ (and hence on $\bar{k}^{\prime}$ ), we have

$$
\frac{d k^{\prime}}{d \tau}=\frac{\partial k^{\prime}}{\partial \tau}+\left[\frac{\partial k^{\prime}}{\partial \tau^{\prime}} \frac{d \tau^{\prime}}{d \bar{k}^{\prime}}+\frac{\partial k^{\prime}}{\partial \ell^{\prime}} \frac{d \ell^{\prime}}{d \bar{k}^{\prime}}+\frac{\partial k^{\prime}}{\partial \bar{k}^{\prime}}\right] \frac{d \bar{k}^{\prime}}{d \tau}
$$


and since $\frac{d \bar{k}^{0}}{d \tau}=\frac{d k^{0}}{d \tau}$, we have

$$
\frac{d k^{\prime}}{d \tau}=\frac{\partial k^{\prime}}{\partial \tau}\left[1-\frac{\partial k^{\prime}}{\partial \tau^{\prime}} \frac{d \tau^{\prime}}{d \bar{k}^{\prime}}-\frac{\partial k^{\prime}}{\partial \ell^{\prime}} \frac{d \ell^{\prime}}{d \bar{k}^{\prime}}-\frac{\partial k^{\prime}}{\partial \bar{k}^{\prime}}\right]^{-1}
$$

We similarly have

$$
\frac{d k^{\prime}}{d \ell}=\frac{\partial k^{\prime}}{\partial \ell}\left[1-\frac{\partial k^{\prime}}{\partial \tau^{\prime}} \frac{d \tau^{\prime}}{d \bar{k}^{\prime}}-\frac{\partial k^{\prime}}{\partial \ell^{\prime}} \frac{d \ell^{\prime}}{d \bar{k}^{\prime}}-\frac{\partial k^{\prime}}{\partial \bar{k}^{\prime}}\right]^{-1}
$$

for the total effect of $\ell$ on $k^{\prime}$ (given some - possibly arbitrary —one-period-ahead policy rules $\ell^{\prime}\left(\bar{k}^{\prime}\right)$ and $\left.\tau^{\prime}\left(\bar{k}^{\prime}\right)\right)$.

The effects given by (36) and (37) will enter the political decision process for $\tau$ and $\ell$. They can be found by differentiating (30), and are detailed in Appendix 1. As one would expect, the direct effect of a transfer from young to old is normally to decrease the saving of the young (i.e. $d k^{\prime} / d \tau<0$ ), while the effect of increased time working relative to accumulating knowledge is to increase saving (i.e. $d k^{\prime} / d \ell>0$ ), assuming that the marginal effect on current earnings is positive, which it always will be at the optimum.

Let $1+\gamma \equiv g(\ell)$ and $q \equiv\left|\frac{d g(\ell)}{d \ell}\right| / g(\ell)$. After some tedious but straightforward manipulations, the first-order conditions for $\left(\mathrm{P}^{\prime}\right)$ turn out to be

$$
\begin{gathered}
\theta \frac{d u\left(c_{2}\right)}{d c}=(1-\theta) \frac{d u\left(c_{2}^{\prime}\right)}{d c} \times \\
\left\{\left(1+f_{1}\left(\bar{k}^{\prime}\right)\right)-\left(1+\gamma^{\prime}\right) \frac{d k^{\prime}}{d \tau}\left[k^{\prime} \ell^{\prime} f_{11}\left(\bar{k}^{\prime}\right)-q^{\prime} \tau^{\prime} \frac{d \ell^{\prime}}{d \bar{k}^{\prime}}+\frac{d \tau^{\prime}}{d \bar{k}^{\prime}}\right]\right\}
\end{gathered}
$$

and

$$
\begin{aligned}
& \theta \frac{d u\left(c_{2}\right)}{d c}\left[(1-q \ell) k\left(1+f_{1}(k)\right)+\tau q\right]= \\
& (1-\theta) \frac{d u\left(c_{2}^{0}\right)}{d c}\left\{( 1 + f _ { 1 } ( k ^ { \prime } ) ) \left\{-\left[(1-q \ell)\left(f(k)-k f_{1}(k)\right)+\tau q\right]-\right.\right. \\
& \left.\left(1+\gamma^{\prime}\right)\left[-q \tau^{\prime}+\frac{d k^{0}}{d \ell}\left(-\tau^{\prime} q^{\prime} \frac{d \ell^{0}}{d k^{0}}+\ell^{\prime} k^{\prime} f_{11}\left(k^{\prime}\right)+\frac{d \tau^{0}}{d k^{0}}\right)\right]\right\}
\end{aligned}
$$


Given $\bar{k}$ and sufficiently well-behaved functions $\tau^{\prime}\left(\bar{k}^{\prime}\right)$ and $\ell^{\prime}\left(\bar{k}^{\prime}\right)$, equations (30)-(32) and (38)-(39) can (in principle) be solved for $\tau$ and $\ell$ as function of $\bar{k}$. The political equilibrium will then be a pair of policy rules $\tau(\bar{k}), \ell(\bar{k})$ such that if $\tau^{\prime}=\tau\left(\bar{k}^{\prime}\right)$ and $\ell^{\prime}=\ell\left(\bar{k}^{\prime}\right)$, then the $\tau$ and $\ell$ values that satisfy (38) and (39), given that $k^{\prime}$ comes from (30)-(32), are $\tau(\bar{k})$ and $\ell(\bar{k})$.

If we combine (38) and (39) to eliminate the marginal utility terms and use the relationship (A3) from Appendix 1 to eliminate $d k^{\prime} / d \ell$ we get (after some simplification)

$$
\begin{gathered}
1-q \ell=\frac{1+\gamma^{\prime}}{1+f_{1}\left(k^{\prime}\right)} \times \\
\left\{q \tau^{\prime}+\left[q \ell k\left(1+f_{1}(k)\right)-\tau q+f(k)+k\right] \frac{d k^{\prime}}{d \tau} \times\right. \\
\left.\left(-\tau^{\prime} q^{\prime} \frac{d \ell^{\prime}}{d \bar{k}^{\prime}}+k^{\prime} \ell^{\prime} f_{11}\left(k^{\prime}\right)+\frac{d \tau^{\prime}}{d \bar{k}^{\prime}}\right)\right\} \times \frac{1}{(f(k)+k)}
\end{gathered}
$$

Recall again that the laissez-faire equilibrium has $1-q \ell=0$, while the optimal steady state has $1-q \ell=(1+\gamma) /\left(1+f_{1}(k)\right)$. The above condition clearly differs from either of these cases, but it is difficult to say much more than that without either simplifying the model or looking at numerical examples. We will take the latter route. See Kahn (1997) for a model without physical capital in which analytical solutions are possible.

\subsection{Numerical Results}

We used polynomial approximation methods (see Appendix 2) to find optimal $\tau(k)$ and $\ell(k)$ functions. Results were computed for a the case of Cobb-Douglas production $f(k)=A k^{\alpha}$ and CES utility $u(c)=c^{1-1 / \sigma} /(1-1 / \sigma)$ under a variety of parametric assumptions. It turns out that relatively low order polynomials (e.g. cubic equations) provide good approximations to the true equilibrium policy rules, at least for $k$ not too small. Figure 2 plots a representative graph of the steady state equilibrium interest rate $1+f_{1}(k)$ and aggregate equilibrium growth rate $g\left(\ell_{e}\right)-1$ against $\theta$. Also plotted are the steady state efficient growth rate $\left(g\left(\ell_{p}\right)-1\right)$ given the same steady-state $k$ 
and the laissez-faire growth rate $g\left(\ell^{*}\right)-1$. (The rates are annualized percentage rates based on a 30 year period). The specific parametric and functional form assumptions are $\alpha=0.3, \sigma=1, A=6$, and $g(\ell)=2 \sqrt{1-\ell^{2}}$. Note that for these parameters the infinite horizon planner's problem is only well-defined for $\theta>0.51$, because the implied social discount rate associated with lower values of $\theta$ would fall below zero, and the maximization problem (P2) would have no solution.

Figure 3 plots the equilibrium and efficient total growth rates $g$ against the interest rate, again in annualized percentage rates. The diagonal dotted line represents the $45^{\circ}$ line, so anything to the left of it is dynamically inefficient. Again the efficient planner's problem is not defined in that region. The equilibrium growth rate here is seen only to kick up in the dynamically inefficient region.

Finally, Figure 4 plots the two equilibrium policy rules $\tau(\bar{k})$ and $\ell(\bar{k})$ for the case of $\theta=0.6$. Note that these policy rules are valid for any value of $\bar{k}$, not just in steady state. At this value of $\theta$ the equilibrium growth rate is 1.34 percent, while the efficient growth rate is 1.75 percent.

The main finding is that for moderate values of $\theta$ (say between 0.5 and 0.7 ) the equilibrium growth rate falls substantially short of the efficient growth rate. This is because the equilibrium growth rate is essentially flat with respect to the interest rate, hence there is no $\bar{k}$ for which the equilibrium growth rate would be efficient. For the case plotted in the figures the equilibrium growth rate hovers at about 1.3 percent all but extreme values of $\theta$, while the efficient rate varies between 1.5 and 2.3 percent. By comparison, the laissez-faire equilibrium growth rate is just under 1.2 percent. Similar results were obtained for a variety of parameters.

The intuition for the qualitative result is that the benefits of growth largely spill over onto subsequent generations. There is no mechanism available by which a subsequent generation can commit to reward the previous generation for its sacrifices. To some extent each generation can extract some reward for growth via its influence on subsequent policy decisions through the state variables of the economy. The government is assumed to exploit this to the extent possible in choosing a point along a 
pseudo-Pareto frontier. In the examples computed this effect is rather meager, and leads to only a slight improvement over laissez-faire.

The other notable feature of the numerical results is that the equilibrium $\ell(k)$ function is virtually flat, and that $\ell$ does not vary much with $\theta$ or with $k$, in or out of steady state. This would appear to rule out explaining differences in growth rates by government preferences across generations (as represented by $\theta$ ). This contrasts with the infinite horizon case in which the social discount rate matters a lot.

\section{Empirical Evidence on Education and Political Stability}

As mentioned earlier, researchers have found that returns to schooling are higher in poorer, low-growth countries. It is straightforward to see that in this model the return to schooling is also higher in the political equilibrium than in the efficient allocation. The usual definition of the return to schooling is the derivative of the log of earnings on years of school. In the model, earnings are $H_{t} \ell_{t}=\bar{H}_{t-1} g\left(\ell_{t}\right) \ell_{t}$. "Schooling" would correspond to $1-\ell_{t}$ and the return to schooling $R_{t}$ (traditionally defined as $d \log ($ earnings $) / d$ schooling) is

$$
R_{t}=\left|g_{1}\left(\ell_{t}\right)\right| / g\left(\ell_{t}\right)-1 / \ell_{t}
$$

This is increasing in $\ell$ over the relevant range (i.e. $0<\ell \leq \ell_{L F}$ ), which implies that $R$ would be higher in the political equilibrium (and higher still under laissez-faire) than under the efficient allocation.

Of course in practice there would have to be exogenous variation in $\ell$ across individuals within a country to produce actual estimates of return to schooling, whereas we have assumed that individuals are identical and all choose the same $\ell$. The point here is that the evidence suggests that there is systematic underinvestment in schooling in low-income/low-growth countries (not just endogenously low investment be- 
cause of a lower payoff), a finding that is consistent with the model. ${ }^{10}$

With this in mind, we briefly examine evidence of a link between education policies and political stability. The data for this exercise come from Barro and Lee (1994), and cover a total of 138 countries over the period 1960-1990. The motivation for looking at political stability is as follows: The model will distinguish between policymakers with an infinite horizon and those with a short horizon. One of the ways a farsighted policymaker could implement an efficient policy is to enact a law that is difficult to undo. That will almost certainly be more difficult to do in an environment of political instability.

The empirical findings should obviously not be considered a direct test of the model. The model is too stylized to match up directly with data. But they do suggest that something like the factors suggested by the model are relevant - the fact that the government does not expect to be around makes it unlikely to set up institutions that achieve precommitment. The measure of instability we use, denoted REVCOUP, is the number of coups and/or revolutions experienced (per year) by each country over the period 1960-1984. We also do not have ideal measures of education policy. We consider three different types of variables: Government expenditures on education as a fraction of GDP (denoted GEXPSH), primary and secondary enrollment rates, and average years of schooling in the population over 25. The latter really measures a stock rather than a flow, but the panel structure of the data enables us to, for example, use this stock as of the end of the time period, as a function of what has occurred in the country over the prior 25 years. On the other hand, only the first really measures something like a government policy variable. Also, GEXPSH to some extent controls for income effects because it is expressed in terms of a share of GDP.

Table I displays statistics on the relationship between the educational variables and the political instability. The enrollment rate variable PSER is a combined primary and secondary enrollment rate, equal to $8 \times$ primary rate $+4 \times$ secondary rate, and has the interpretation of number of years or primary education (out of 12) the current school-age population is receiving on average. The variable YS85 is average years of 
schooling in the over 25 population as of 1985. All three education variables are significantly negatively correlated with political instability. The table also examines conditional means of the education variables based on whether REVCOUP $=0$ or REVCOUP $>0$. The conditional sample means differ by economically meaningful amounts.

Of course these facts could be explained entirely by the fact that both education and political stability are positively related to wealth or income. Even government expenditure on education could have an income elasticity significantly greater than one, which could account for the negative correlation of GEXPSH and political stability. To explore this possibility, Table II reports regression results of the education variables on a constant, $\log (\mathrm{GDP}$ ) (where GDP is averaged over 1960-1990), and REVCOUP. Similar results obtained when REVCOUP was replaced with a dummy variable equal to one when REVCOUP $>0$, zero otherwise. Similar results also obtained for regressions run separately for each time period in the sample (e.g. 1980-85 GEXPSH on $1980 \log ($ GDP) and REVCOUP). Two sets of results are shown: Least squares weighted by 1960 population, and ordinary least squares.

The differences between the weighted and unweighted results suggest that a number of very small countries add a lot of noise to the OLS results, at least for PSER and YS85. But overall the results point strongly to a negative impact of political instability on educational attainment even after controlling for income level. The interpretation of the WLS results, for example, is that a country experiencing one coup or revolution per year (and there are such countries in the Barro-Lee data set) would have government expenditures on education as a share of GDP smaller by 2.1 percentage points (which is on the order of 50 percent of the mean!) than a politically stable country with the same GDP. The average years of schooling for the over age 25 population would be smaller by 2.7 years, and average primary-secondary enrollments would be smaller by about 3.3 years.

An alternative explanation of these facts is that education is simply less productive in poorer or less politically stable countries. As noted earlier, however, researchers 
have found that less developed countries have significantly higher returns to schooling than developed countries (see Psacharopolous (1973)). ${ }^{11}$ The explanation offered in the model is that the high returns in those countries reflect endogenous policy decisions not to encourage human capital accumulation to the same extent as in developed countries. Those decisions in turn reflect a lack of incentive on the part each current generation to accumulate human capital when the benefit falls primarily on subsequent generations, together with the lack of stable political institutions that can achieve the desired intergenerational cooperation.

Finally, it is possible that there is reverse causality, i.e. that suboptimal education policies lead to political instability. To deal with this econometrically we would need instruments for REVCOUP that are correlated only with the "exogenous" component of political instability, i.e. the part that is not related to this reverse effect. Although this is beyond the scope of the current paper, variables related to instability from external sources or geography could serve this purpose.

\section{Conclusions}

When individuals have finite horizons, the market may not provide sufficient reward for actions whose benefits spill over onto future generations. In this paper, a "representative" government (i.e. one whose preferences reflect those of its constituents) endowed merely with the ability to tax and transfer can improve somewhat on the market allocation, but cannot achieve first-best growth. Efficiency requires in addition the ability to bind future governments. We argue that this ability is related to political stability, and provide empirical evidence that stability and growth-related policies (namely education) are meaningfully related. If developed countries have overcome this problem, it must be the result of stable institutions that outlast whoever happens to be in power - in particular institutions that either allow those who accumulate human capital to recoup more of the benefits (via intergenerational transfers), or that simply mandate such accumulation. 
Some recent literature has focused on intra-generational conflict ${ }^{12}$, as a result of which the political system potentially makes incorrect decisions regarding investment in human capital because of heterogeneous preferences. That story would not predict a link between political instability and education policy, unless the heterogeneity provoked instability. More closely related recent literature would include Tabellini and Alesina (1990), who show how intergenerational conflict (plus the inability to precommit) leads to inefficient fiscal policy, and Cukierman et al. (1992), who have a model and empirical results linking the use of seignorage with political instability. ${ }^{13}$

Other familiar models have similar concerns with the incentives of future generations. In monetary models (e.g. Samuelson (1958)) each young generation's willingness to accept money for goods is dependent on their belief that the subsequent generation will accept it from them. In the capital accumulation model each young generation's willingness to transfer wealth to the old is dependent on their belief that the same thing will happen in the subsequent time period.

We have limited our attention to Markovian solutions, i.e. those in which policies only depend on the state of the economy. ${ }^{14}$ While this ignores potential historydependent equilibria that involve (for example) trigger strategies, the Markovian solutions have the virtue of being renegotiation-proof. We also have assumed a certain level of sophistication by the decision-maker in anticipating the impact of current policy choices on future policies, as opposed to naively taking future policies as given. The naive behavior is analogous to the Cournot assumption in models of imperfect competition, where each producer takes the others' quantities as given in its own quantity decision. The sophisticated behavior corresponds to the Stackelberg assumption that one producer can act first and take the others' responses into account. We would argue that the sophisticated behavior is a priori the preferred assumption. $^{15}$ In any case, neither the naive nor the sophisticated behavior brings about efficiency. Only full cooperation or precommitment accomplishes that. 


\section{Appendix 1: Model Solution}

Each of the components of 36 and 37 can be found by total differentiation of 30. After converting second derivatives of utility into relative risk aversion, and substituting 30 in various places, we get

$$
\begin{gathered}
\left(1+\gamma^{\prime}\right) \frac{d k^{\prime}}{d \tau}=-\varphi^{\prime} / \\
\left\{\left(1+f_{1}\left(k^{\prime}\right)+\varphi^{\prime}\right)\left(\ell^{\prime}+\left(1-q^{\prime} \ell^{\prime}\right) k^{\prime} \frac{d \ell^{\prime}}{d \bar{k}^{\prime}}\right)-\right. \\
\left.\tau^{\prime} q^{\prime} \frac{d \ell^{\prime}}{d \bar{k}^{\prime}}+\frac{d \tau^{\prime}}{d \bar{k}^{\prime}}+f_{11}\left(k^{\prime}\right)\left[\ell^{\prime} k^{\prime}-\sigma\left(\ell^{\prime} k^{\prime}+\tau^{\prime} /\left[1+f_{1}\left(k^{\prime}\right)\right]\right)\right]\right\}
\end{gathered}
$$

and

$$
\begin{gathered}
\left(1+\gamma^{\prime}\right) \frac{d k^{\prime}}{d \ell}= \\
\varphi^{\prime}\left((1-q \ell)\left(f(k)-k f_{1}(k)\right)+q\left[g\left(\ell^{\prime}\right) \ell^{\prime} k^{\prime}+\tau\right]\right)+ \\
\left(1+\gamma^{\prime}\right) q\left[\ell^{\prime} k^{\prime}\left(1+f_{1}\left(k^{\prime}\right)\right)+\tau^{\prime}\right] / \\
\left\{\left(1+f_{1}\left(k^{\prime}\right)+\varphi^{\prime}\right)\left(\ell^{\prime}+\left(1-q^{\prime} \ell^{\prime}\right) k^{\prime} \frac{d \ell^{\prime}}{d \bar{k}^{\prime}}\right)-\right. \\
\left.\tau^{\prime} q^{\prime} \frac{d \ell^{\prime}}{d \bar{k}^{\prime}}+\frac{d \tau^{\prime}}{d \bar{k}^{\prime}}+f_{11}\left(k^{\prime}\right)\left[\ell^{\prime} k^{\prime}-\sigma\left(\ell^{\prime} k^{\prime}+\tau^{\prime} /\left[1+f_{1}\left(k^{\prime}\right)\right]\right)\right]\right\}
\end{gathered}
$$

where $1+\gamma=g(\ell), q=\left|\frac{d g(\ell)}{d \ell}\right| / g(\ell)$, and $\varphi=\left[\left(1+f_{1}(k)\right) /(1+\beta)\right]^{\sigma}$. Further manipulations of the numerator of (A2) (using the fact that $\varphi^{\prime}$ also equals $c_{2}^{\prime} / c_{1}$ ) lead directly to the result that

$$
\frac{d k^{\prime}}{d \ell}=-\left[f(k)-k f_{1}(k)\right] \frac{d k^{\prime}}{d \tau} .
$$




\section{A ppendix 2: Numerical Solutions}

This appendix describes how we solve for the equilibrium policy rules $\tau(k)$ and $\ell(k)$ numerically. We assume that the functions can be approximated by a polynomial. Specifically we will assume that

$$
\begin{aligned}
& \tau(x)=\sum_{i=0}^{m} v_{i} p_{i}(x) \\
& \ell(x)=\sum_{i=0}^{m} \omega_{i} p_{i}(x)
\end{aligned}
$$

where $p_{i}$ is the ith-order Chebyshev polynomial. The Chebyshev polynomials are a family of orthogonal polynomials defined by $p_{0}(x)=1, p_{1}(x)=x, p_{i}(x)=2 x p_{i-1}(x)-$ $p_{i-2}(x)$, on the interval $[-1,1]$. To implement this in the current context it is necessary to do a change of variables in the system (38)-(39) so that it can be expressed in terms of a variable $x$ whose domain is $[-1,1]$.

If $\tau(x)$ and $\ell(x)$ satisfy the above, then $d \tau / d x$ and $d \ell / d x$ are defined accordingly. The solution procedure involves selecting a value of $m$ and finding values of $\left\{v_{i}\right\}$ and $\left\{\omega_{i}\right\}$ that approximately satisfy the system (38)-(39). A variety of methods can be used to find solutions that are good approximations. One convenient method advocated by practitioners of numerical techniques (e.g. Judd (1991)) is to solve the system exactly at $m+1$ points, specifically the roots of $p_{m+1}$. The accuracy of the fit can than be checked at intermediate points, and in particular at the value of $x$ that corresponds to the steady state value of $\bar{k}$. Then do the change of variables in reverse to get $\tau$ and $\ell$ in terms of $\bar{k}$. Thus finding a solution becomes a matter of solving $2(m+1)$ equations for $2(m+1)$ unknowns (since we are solving for two functions), where $m$ can easily be less than 5 , as compared to grid search methods that require solving the system at potentially hundreds of values of $k$. 


\section{Footnotes}

${ }^{1}$ See, for example, Alesina et al. (1996).

2 Azariadis and Drazen (1990) explore different issues with a similar extension of the Diamond model.

3 Of course in reality some knowledge is excludable. All that is required for the model is that some knowledge not be inter-generationally excludable. Intra-generational excludability is just a simplifying assumption.

${ }^{4}$ Some stronger condition on $\rho$ is required to ensure that the objective is finite. A more than sufficient condition is $1+\rho>g(0)$.

5 Majority voting would not be very interesting in this context with only two types of agents.

6 Although some types of intergenerational altruism in which agents effectively have an infinite horizon - such as in Barro (1974) — would make this problem completely uninteresting, the results that follow are not sensitive to the inclusion of a conventional bequest motive.

7 The government's preferences in this problem and in the earlier infinite horizon specification are limiting cases of the dynamically inconsistent quasi-hyperbolic preferences analyzed (and attributed to individuals) by Laibson (1996), and earlier by Phelps and Pollak (1968). The more general case would involve the objective $\left[u\left(c_{1 t}\right)+\frac{1}{1+\alpha} u\left(c_{2, t+1}\right)\right]+\frac{1+\rho}{1+\alpha} u\left(c_{2 t}\right)+\beta \sum_{i=1}^{\infty}(1+\rho)^{-i}\left[u\left(c_{1, t+i}\right)+\frac{1}{1+\alpha} u\left(c_{2, t+i+1}\right)\right]$, where $0 \leq \beta \leq 1$. This paper only examines the cases $\beta=0$ and $\beta=1$. Analysis of the general case would be an interesting subject for future research.

8 If $k$ were defined as $K / H_{-1}$, for example, all of the derivations below would end up with first, second, and cross-partial derivatives of $F$, which in turn would involve $k /(g(\ell) \ell)$.

9 We will adopt the usual practice of letting, for example, $d \tau^{\prime} / d \bar{k}^{\prime}$ refer to the first derivative of $\tau^{\prime}(\cdot)$ evaluated at $\bar{k}^{\prime}$.

10 Note that in the model the return actually equals zero for the laissez-faire choice 
of $\ell$, and is negative for $\ell_{e}$ and $\ell^{*}$. This is simply because the private decision problem for $\ell$ is essentially static, and there is no direct cost of schooling. It would be easy to modify the model to generate positive returns.

11 Ljungqvist (1995) suggests a second-best insurance explanation for this stylized fact.

12 See, for example, Fernandez and Rogerson, 1995, Glomm and Ravikumar, 1992, and Saint-Paul and Verdier, 1993.

13 See also Persson and Svensson (1989), Persson, Persson, and Svenson (1987), Cukierman and Meltzer (1989).

14 See, for example, Kotlikoff, Persson, and Svensson (1988).

15 Persson and Svensson (1989), for example, rely on such an assumption in modelling a government's fiscal stance. 


\section{R eferences}

Alesina, A., S. Özler, N. Roubini, P. Swagel (1996), "Political Instability and Economic Growth," J ournal of E conomic Growth, 1, 189-211.

Azariadis, C. and A. Drazen (1990), "Threshold Externalities in Economic Development," Quarterly J ournal of E conomics, 105, 501-526.

Barro, R. (1974), "Are Government Bonds Net Wealth?" J ournal of Political Economy, 82, 1095-1117.

Cass, D. (1972), "On Capital Overaccumulation in the Aggregate, Neoclassical Model of Economic Growth," J ournal of Economic Theory, 4, 200-223.

Cukierman, A. and A. Meltzer (1989), "A Political Theory of Government Debt and Deficits in a Neo-Ricardian Framework," American E conomic Review, 79, 713-732.

Cukierman, A., S. Edwards, and G. Tabellini (1992), "Seigniorage and Political Instability," A merican E conomic Review, 82, 537-555.

Diamond, P. (1965), "National Debt in a Neoclassical Model," American Economic Review, 55, 1126-1150.

Fernandez, R. and R. Rogerson, (1995) "On the Political Economy of Education Subsidies," Review of E conomic Studies, 62, 249-262.

Glomm, G. and B. Ravikumar, (1992) "Public and Private Investment in Human Capital: Endogenous Growth and Income Inequality," J ournal of Political Economy, 100, 818-834.

Judd, K. (1991), "Minimum Weighted Residuals Methods for Solving Dynamic Economic Models," Hoover Institution, manuscript.

Kahn, J. (1997), "Education, Poltical Instability, and Growth" Federal Reserve Bank of New York Research Paper \#9737. 
Kotlikoff, L., T. Persson, and L. Svensson (1988), "Social Contract as Assets: A Possible Solution to the Time Consistency Problem," American E conomic Review, $78,662-667$.

Laibson, D. (1996), "Hyperbolic Discount Functions, Undersaving, and Savings Policy," National Bureau of Economic Research, Working Paper \#5635.

Lucas, R. (1988), "On the Mechanics of Economic Development," J ournal of Monetary E conomics, 22, 3-42.

Persson, M., T. Persson, and L. Svensson (1987), "Time-Consistency of Fiscal and Monetary Policy," E conometrica, 55, 1419-1432.

Persson, T. and L. Svensson (1989), "Why a Stubborn Conservative Would Run a Deficit: Policy with Time-Inconsistent Preferences," Quarterly J ournal of Economics, 104, 325-346.

Phelps, E., and R. Pollak (1968), "On Second-best National Saving and Gameequilibrium Growth," Review of Economic Studies, 35, 185-199.

Psacharopolous, G. (1973), Returns to E ducation: An International Comparison, San Francisco: Elsevier.

Saint-Paul, G. and T. Verdier (1993), "Education, Democracy, and Growth," J ournal of Development Economics, 42, 399-408.

Samuelson, P. (1958), “An Exact Consumption Loan Model of Interest with or without the Social Contrivance of Money," J ournal of P olitical E conomy, 66, 1002-1011.

Tabellini, G., and A. Alesina (1990), "Voting on the Budget Deficit," A merican Economic Review, 80, 37-49.

Uzawa, H. (1965), "Optimal Technical Change in an Aggregative Model of Economic Growth," International E conomic Review, 6, 18-31. 
Table I: Education Variables and Political Instability ${ }^{a}$

\begin{tabular}{cccc}
\hline & PSER & YS85 & GEXPSH(\%) \\
\hline Correlation(REVCOUP, $\cdot)$ & -0.339 & -0.336 & -0.337 \\
$E\{\cdot \mid$ REVCOUP $>0\}$ & 6.993 & 4.290 & 3.370 \\
$E\{\cdot \mid$ REVCOUP $=0\}$ & 9.925 & 7.723 & 4.789 \\
\hline
\end{tabular}

${ }^{a} \mathrm{REVCOUP}$ is the number of revolutions or coups per year.

PSER is the combined primary and seconary enrollment rate.

Y S85 is the average years of schooling for the over-25 population as of 1985.

GEXPSH is government expenditures on education as a fraction of GDP 
Table II: Education Variable Cross-Section Regression Results ${ }^{a}$

\begin{tabular}{lcccc}
\hline \multicolumn{5}{c}{ WLS Results } \\
\hline & $\log ($ GDP $)$ & REVCOUP & $R^{2}$ & \#obs \\
\hline PSER & 1.384 & -3.275 & 0.996 & 98 \\
& $(0.050)$ & $(0.544)$ & & \\
YS85 & 2.549 & -2.720 & 0.971 & 98 \\
& $(0.091)$ & $(1.052)$ & & \\
GEXPSH & 0.0090 & -0.021 & 0.983 & 89 \\
& $(0.0004)$ & $(0.005)$ & & \\
\hline \hline \multirow{5}{*}{ OSLS Results } & & \\
\hline PSER & $\log ($ GDP $)$ & REVCOUP & $R^{2}$ & $\#$ obs \\
& 2.302 & -0.388 & 0.738 & 98 \\
YS85 & $(0.149)$ & $(0.589)$ & & \\
& 2.455 & -1.102 & 0.759 & 98 \\
& $(0.154)$ & $(0.607)$ & & \\
GEXPSH & 0.0066 & -0.013 & 0.321 & 89 \\
& $(0.0014)$ & $(0.005)$ & & \\
\hline
\end{tabular}

${ }^{a}$ See note to Table I for variable definitions. 
Figure 3: Equilibrium and Efficient Growth and Real Interest Rates

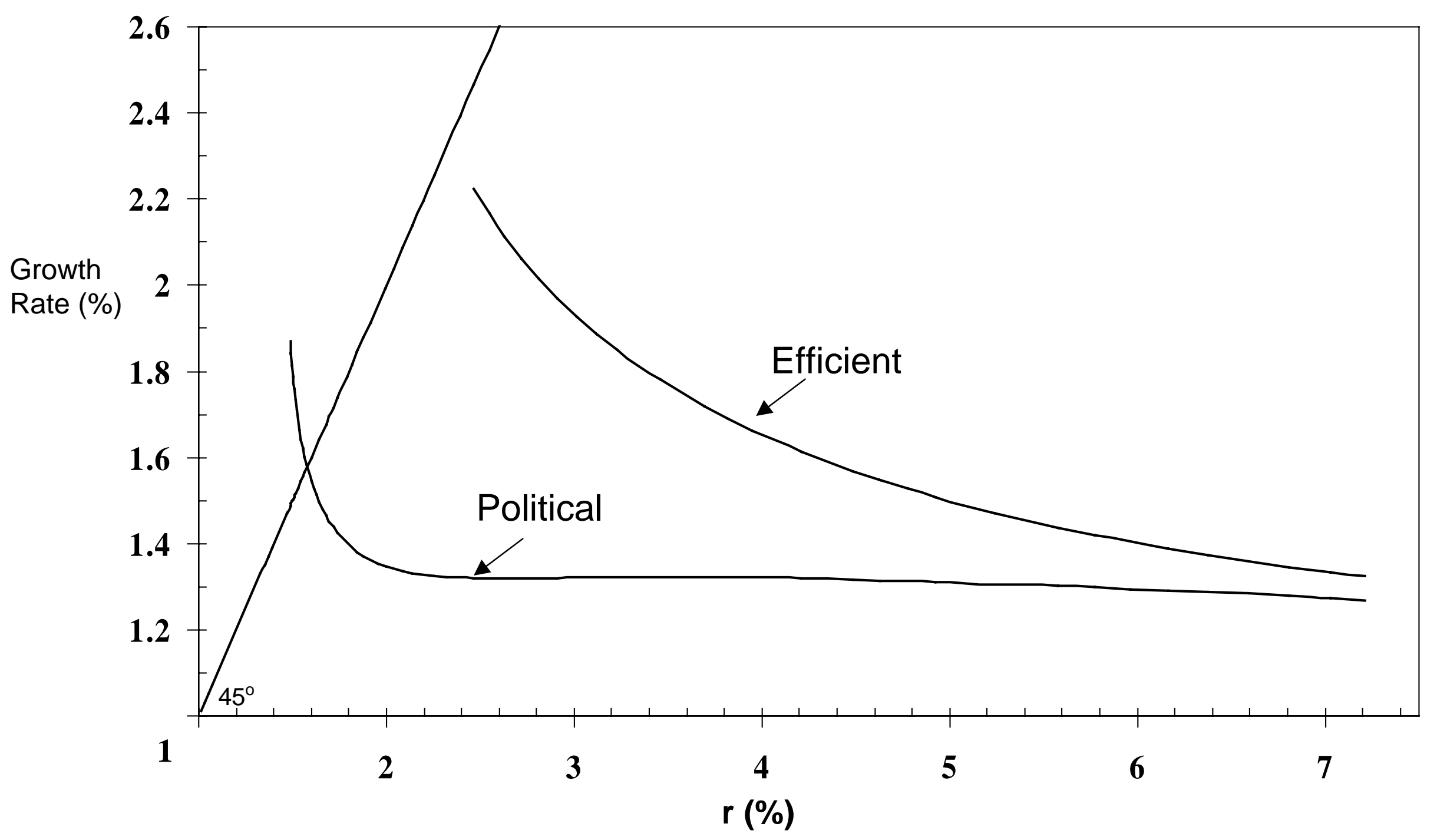


Figure 4: Equilibrium Policy Rules

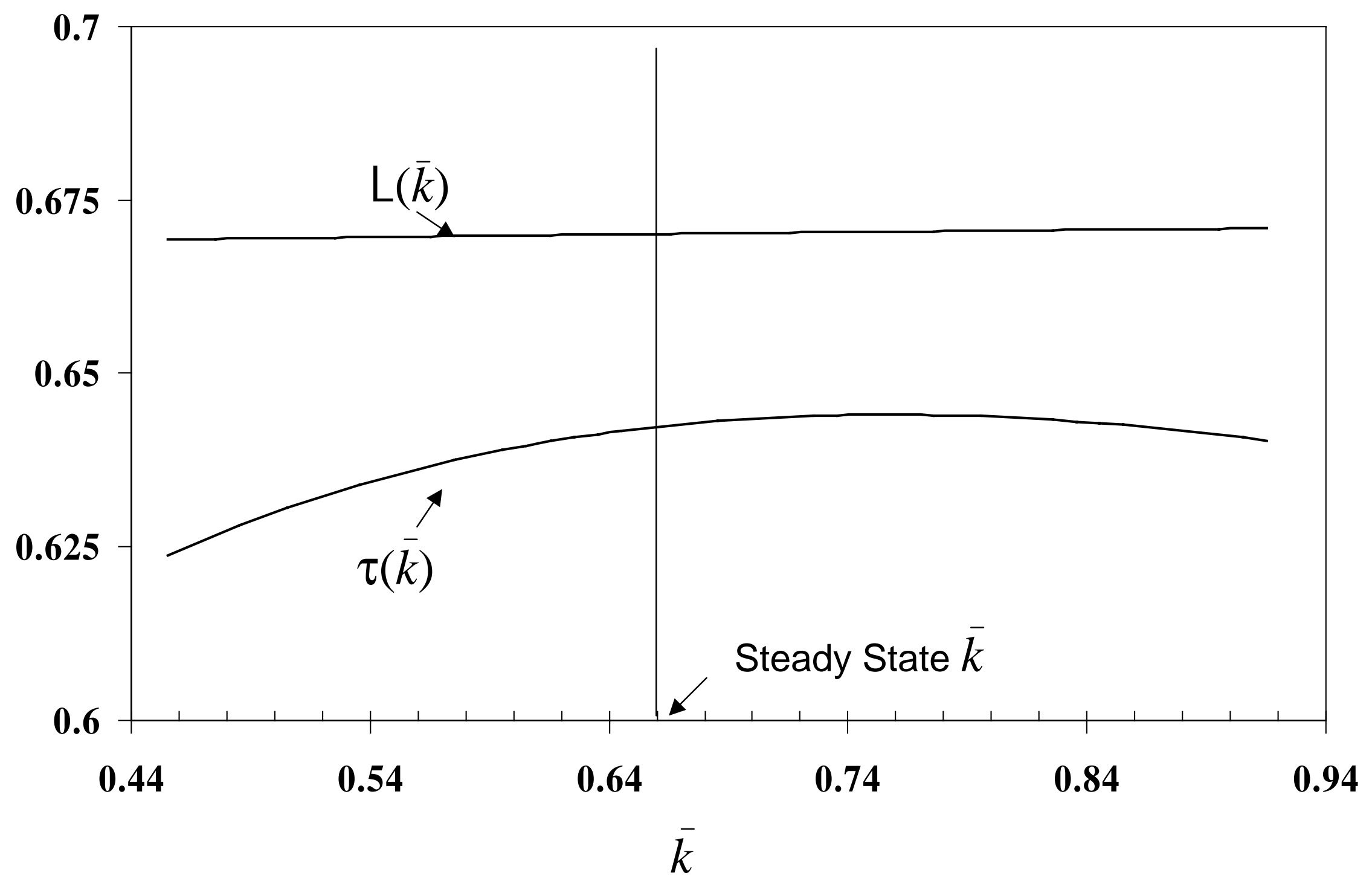

